\title{
LA COCA EN EL INTERCAMBIO MERCANTE ATLÁNTICO-MEDITERRÁNEO ${ }^{1}$
}

\author{
THE “COCA” IN THE MERCHANT ATLANTIC-MEDITERRANEAN EXCHANGE
}

\author{
ANTONIO ORTEGA VILLOSLADA \\ Doctor en Historia. UNED
}

\begin{abstract}
Resumen: Se aborda el paso de la coca al Mediterráneo y su relevancia en la náutica medieval. Primacía determinada por el timón de codaste, conocido genéricamente por los marinos de la cuenca mediterránea como bayonés, es decir, del Golfo de Vizcaya, cuyos patrones arribarán regularmente a Mallorca
\end{abstract}

Palabras clave: Mallorca; Atlántico; Comercio naval; Buques; Coca.
Abstract: One approaches the passage of the coca to the Mediterranean and its relevancy in the medieval navigation. Superiority determined by the rudder of sternpost, know generically by the sailors of the mediterranean basin as bayonés, that is to say, of the Gulf of Biscay, whose skippers will arrive regularly to Majorca.

Keyword: Majorca; Atlantic; Naval trade; Ships; Coca.

\section{SUMARIO}

1. Evolución.-2. Áreas de navegación.- 3. Timón bayonés.- 4. Arribada de patrones del Golfo de Vizcaya a Mallorca.

El término $\operatorname{coca}^{2}$ deriva de la palabra latina concha. En alemán es nombrada como kogge, resultante del germánico kok - concha-; afinidad que procura su configuración de alto bordo, corta eslora y amplia manga - estructurada en una o varias cubiertas-. Para Carbonell Relat ${ }^{3}$ podría tener raíz semítica, pues en el islam se dió un tipo de buque de poca eslora y muy mangudo llamado kuggi o kuggr, nombre relacionado con el de koggi - tonel en árabe-. Las noticias retrotraen a los Annales Bertiani -año 867- en los

\footnotetext{
${ }^{1} \mathrm{El}$ presente texto proviene de mi tesis El Reino de Mallorca y el mundo atlántico (12301349), en la que analizo las relaciones del conjunto de territorios de la Casa de Mallorca con el ámbito oceánico.

Las siglas utilizadas en este artículo son: ARM = Archivo del Reino de Mallorca; $\mathrm{AH}=$ Archivo Histórico; RP = Real Patrimoniop; LR = Letras Reales; ACM = Archivo Capitular de Mallorca; $\mathrm{AHCB}=$ Archivo Histórico de la Ciudad de Mallorca; PRO = Public Record Office

${ }^{2}$ Buque de variada grafía: coca, choca, cocha, coqua, cocqua, qoqa, cog, coga, coha, koga, kogge, cogue, coque, coke, coge, cochis, coggis, kogcho, kocho, quoche, choque...

${ }^{3}$ Laureano CARBOnell Relat, La "Coca", nave del Medioevo, "Revista de Historia Naval", IV/15 (1986), p. 49.
} 
que el pueblo frisón es citado bajo el apelativo de cokingi (gente de coca). No obstante, las primeras alusiones como tal embarcación son del siglo XII con los vocablos kogcho y kocho.

Dedicadas al comercio y a la guerra, actualmente no se discute su procedencia atlántica aunque si su expansión y evolución ${ }^{4}$. Se introducen - siglo XIII- en el Mediterráneo ${ }^{5}$ y pronto adoptan el sistema constructivo de este mar haciéndose con el forro a tope y equipadas con un único mástil que, a principios del XIV, se verá completado con un rudimentario bauprés, armando - ya en el siglo XV- tres mástiles. El arqueo ${ }^{6}$ se sitúa entre las veinte toneladas de las cocas de un puente y las cuatrocientas para las de tres puentes.

En la década de los noventa se produjeron investigaciones relevantes respecto a su comportamiento, gracias a los afortunados hallazgos de la coca

4 "La nave que se conocía por este nombre -coca, cog, kogge-en el Atlántico Norte en estos años (inicios del siglo XIV) era una embarcación de porte de 50 a 80 toneles, con timón de codaste, un mástil, un solo puente y un castillo de popa muy discreto o casi inexistente, forrada a tingladillo, de fondo plano con una zapata en lugar de quilla, una gran vela cuadrada y un bauprés. Lo más característico era la disposición de la roda y el codaste, que se disparaba de la zapata en línea recta y daba al buque su típica silueta rígida. Su eslora media era de 15 a $20 \mathrm{~m}$ ". E. FERREIRA PRIEGUE, Galicia en el comercio marítimo medieval, La Coruña, Fundación "Pedro Barrié de la Maza", 1988, p. 243. Capmany halla referencias desde mediados del siglo XI en diversas crónicas: “....Este género de construcción se inventó probablemente en el Océano, pues en Matheo de Westminster, Matheo de Paris y Thomas WALSINGHAM se hace mención de estos baxeles desde el año 1066, como propios de los Normandos, y después de los ingleses y otros". Antonio de CAPMANY Y DE MONPALAU, Memorias históricas sobre la Marina, Comercio y Artes de la antigua Ciudad de Barcelona, Barcelona, 1961-1963, I, p. 53, nota 6.

${ }^{5}$ Entran en el Mediterráneo formando parte de las distintas flotas de cruzados que en el curso de los siglos XII y XIII transfretan el estrecho de Gibraltar para marchar sobre Tierra Santa. Desarrollo este punto en Viajes a Flandes e Inglaterra. ¿Cabotaje o recta via?, "Espacio, Tiempo y Forma", Serie III, Historia Medieval. Tomo 16 (2003), pp. 229-249.

${ }^{6}$ Existía un tipo de buque conocido como cocheta. Se registra a partir de 1332 y los documentos indican que se trataría de una embarcación de escasa entidad con las características propias de las cocas: "...que en lo port de Marseyla hauia lexada huna Galea de Janoueses Guelfos robadors, la qual Galea hauia presa una Cocheta de catalans en les mars de Cap de boch; he hauia entes lo dit leny que la dita Galea fasia la via de Catalunya per robar". ARM, AH-4384, f. $84 \mathrm{v}$. (año 1340). .... Berardi banyarons... qui est de barchinona ... captus fuit... cum quadam cocheta... ARM, Suplicaciones no 15 . f. 47 r. (año 1342). En 1341 se construirá una cocheta bayonesca de una cubierta en el litoral de Palma, en los terrenos del antiguo cementerio judío: "...Petrus Pontironi, ciuis Maioricarum, dominus et patronus cuiusdam Cochete bayonesie unius cohoperte uocate Sanctus Nicholaus quam ad huc feri et construi facio iuxta fossare anticum judeorum, confiteor uobis Philipo Marchoni, ciuis Maioricarum..." ACM, Protocolos notariales $\mathrm{n}^{\circ}$ 14564, ff. 133 v-134 r. Poco después Antonio Junus, dueño de la mitad de la coca bayonesa de una cubierta San Vicente, vende 1/16 de la misma y en el documento se habla de esta coca como una cocheta: “...Anthonius Junius, ciuis Maioricarum, dominus per dimidia cuiusdam coche bayonesie unius cohoperte vocatus Sanctus Vicencius nunc existens in portu Maioricarum, quas ducit et patronizat Ffranciscus Roberti, ciuis Maioricarum, confiteor vobis Guillelmo Cardona, ciuis Maioricarum, et vestris quod habetis et habere debetis in dicta mea dimidia dicte cochete et in omnibus exarciis et aparatibus eiusdem..." Ibidem, ff. 137 r-138 r. En 1340 Francisco Roberto y Pedro Serra, patrones de la cocheta San Vicente, realizan un viaje a Túnez: ...patronis unius cochete vocata Sent Vicens, quod possit facere et complire viagium... ARM, ÄH-439, f. 169 r. En 1342 llegan a las Baleares dos cochetas con grano procedentes de la Península. Una de ellas aparece en aguas de La Dragonera, ordenando el lugarteniente, en virtud de la guerra abierta entre Pedro IV y Jaime III, que la apresen y la conduzcan a la ciudad: "Con nos aiam entes de cert que en les mars dela palomera es aplecada una Cochata carregada de forment, dela qual es patro en botet e un companyo seu, diem eus manam..." ARM, AH-3, f. 90 V. La segunda es capturada cerca de Menorca. Ibidem, f. 176 r. El ancoraje de 1332 refleja la presencia de la coqueta del pisano Juan Delpont: "Disapte XVIIII yorns de deembre, Reebem den Johan Delpont, Pisa, per ancoratge dela sua coqueta..........VIIIs". ARM, RP-1100, f. $10 \mathrm{v}$. 
alemana de Bremen y de la holandesa de $\mathrm{Kampen}^{7}$, ambas de la segunda mitad del XIV. La reconstrucción a tamaño real de sendas réplicas ha posibilitado la realización de pruebas rigurosas sobre sus cualidades náuticas ${ }^{8}$. La coca de Bremen' tenía una superficie vélica de $200 \mathrm{~m}^{2}$, eslora máxima de 23 '3 metros, eslora en la quilla de 15'60, manga de 7'6 metros y puntal de 3'14 metros. El calado - a plena carga- era de 2'25 metros y la bodega de $150 \mathrm{~m}^{3}$. La experimentación con las reproducciones ha dado como resultado una velocidad media máxima de seis nudos con vientos de fuerza $6^{10}$. Los primeros ensayos, en 1991 y 1992, demostraron, en contra de lo conjeturado hasta la fecha, su facilidad para navegar y capacidad de maniobra, aun con vientos de través, así como gran estabilidad bajo fuerte temporal ${ }^{11}$.

Estaban provistas de castillos a proa y popa protegidos, en caso de guerra, con paveses de mimbre recubiertos de cuero o falcas de madera, desde donde se combatía al enemigo. Análoga función desempeñaba la cofa durante los enfrentamientos ${ }^{12}$.

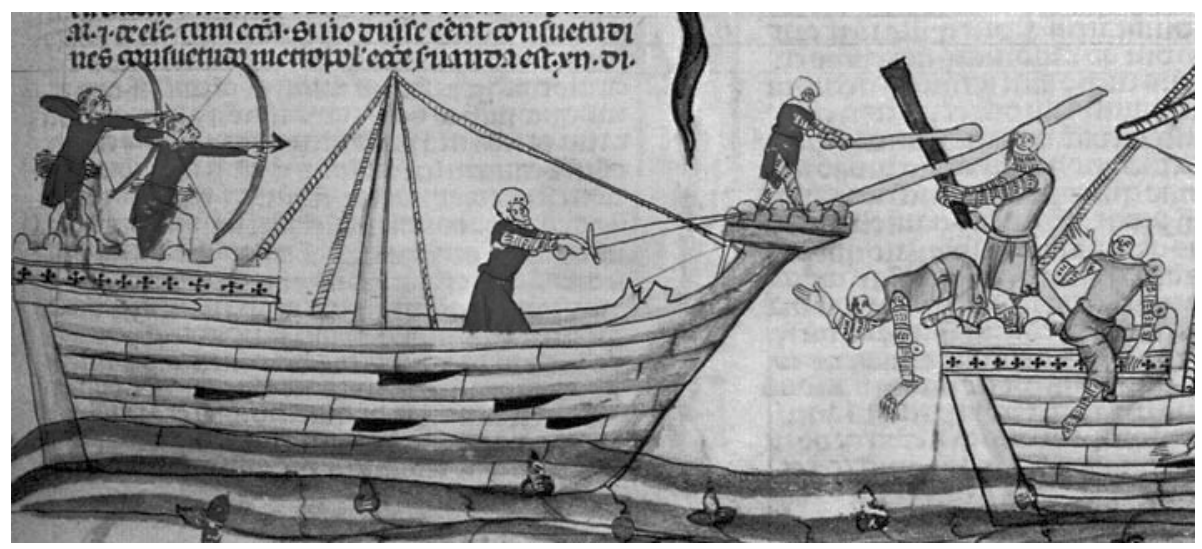

Combate naval entre dos cocas (s. XIV). Smithfield Decretals. British Library, Londres. Hs. Roy. 10. E. IV, fol. 19

${ }^{7}$ En el año 2000 se localizó un nuevo pecio en Doel. Die Kogge, Sternstunde Sternstunde der deutschen Schiffsarchäologie. Schriften des Deutschen Schiffahrtsmuseums. Ediciones Convent. 2003 p. 139.

${ }^{8}$ El Museo Alemán de la Marina ha publicado el resultado de los estudios llevados a cabo durante más de una década con la réplica de la coca de Bremen: Gabriele HoFFMANN y Uwe SCHNALL, Die Kogge, cit. Puede verse una breve aproximación en: www.hansekogge.de. Para la coca de Kampen: www.kamper-kogge.nl

${ }^{9}$ Las medidas de la coca de Kampen son muy similares a la de Bremen.

${ }^{10} \mathrm{Con}$ vientos de fuerza tres navegan a unos 3'5 nudos; con fuerza cuatro a unos 4 nudos y con fuerza cinco a algo más de 5 nudos.

${ }^{11}$ Die Kogge, Sternstunde..., p. 26.

${ }^{12}$ L. CARBOnell Relat, La “Coca”, nave, cit., p. 46. 


\section{EVOLUCIÓN}

Cerca de Utrecht, en Dorestad, se hallan las primeras representaciones de una coca, en monedas de plata del siglo IX, siendo preciso esperar a principios del XII para encontrar, en el ámbito de la Hansa, referencias escritas $^{13}$

Tanto en Inglaterra como en la zona centrada entre la Gascuña y Flandes existen múltiples menciones de la primera mitad del XIII ${ }^{14}$. En 1206 está contextuada en el Báltico. En Portugal se señala en 1297 como uno de los tipos navales del Cantábrico y golfo de Vizcaya surtos en el puerto de Lisboa $^{15}$.

Debido a los contactos comerciales de la costa septentrional peninsular con los mercados noratlánticos, en Castilla su conocimiento se podría remontar al siglo XII, aun no habiendo quedado registrada en sus costas. Para algunos historiadores la primera noticia está en Las Partidas, si bien las distintas redacciones, según la edición consultada, dificultan asegurar este punto $^{16}$. Es, sin embargo, en la Corona de Aragón -y en el Mare Nostrum en general- donde el término coca es asimilado plenamente por la gente del mar. Las citas más antiguas del paso al Mediterráneo surgen en textos de autores de extracción no mediterránea, de finales del XII e inicios del XIII ${ }^{17}$, por lo común ligados a expediciones que desde los mares del Norte se dirigen

\footnotetext{
${ }^{13}$ Ibídem, p. 51.
}

${ }^{14}$ En 1223 cuatro naves -nefs- de Bayona y una coca transportan vino de Aunis a Portsmouth. GOYHENECHE, Bayonne et la règion Bayonnaise du XII ${ }^{e}$ au XV $V^{e}$ siècle, p. 368. En los Rotuli Litterarum. Clausarum in Turri Londinensi... de 1206 se hace referencia al asalto de una coca: "...captos in coga que debuit ire namiata..."; Close Rolls -Henrique III, 1227-1229-, p. 290 -año 1229-: "Quia Ferrandus comes Flandrie et burgenses de Bruges... fuerunt in coga que venit de Cornubia et qui arestati fuerunt eo quod credebatur eos esse de Rupella..."; IDEM, p. 306 -año 1230-: "...quod cogam de Bruges que vocatur Burdernara quam arestare fecit in portu de Sandwich..."; Rôles Gascons, Fragmentus Rotuli Vasconiae, doc. 279 -1242-: "Gerardus de Stavere qui ducit cogam que vocatur legerepe, habet litteras de conducț, scilicet, quod ipse cum coga illa salvo et secure venire possit in terram et potestatem Regis...

${ }^{15}$ Descobrimentos Portugueses. Documentos para a sua história publicados e prefaciados por João MARTINS DA SILVA MAROUES, vol. I (1147-1460) -complemento-,Lisboa, Edič̃o do Instituto para a Alta Cultura, 1944, pp. 21-25: “...como recresesse tençom e peleia e uolta entre o Conçelho da Cidade de Lixboa da hua parte e os Maestres, Mercadores, Marieyros e outras gentes que andam nas naues e nos Baixees e nos Aloques e nas Cocas de Ffonte Rabia de Sam Seuaschaao, de Vermeeo, de Quitari, de Crasto, de Laredo, de Santamder e de Abelhes e da Crunha que ora estam no Porto da dita Cidade de Lixboa..."

${ }^{16}$ Tanto Eberenz como Carbonell Relat extraen los datos de una edición de 1807 en la que figura la coca como uno de los buques castellanos; sin embargo, la que utilizo para este trabajo (impresa por Gregorio López en 1555) no la incluye, pero sí el término carraca. La noticia más antigua que he localizado está datada en 1297, merced a un pleito promovido en Lisboa. En 1303 se mencionan en los archivos ingleses algunas cocas gallegas en Burdeos, pero de nuevo el texto no está redactado en la Corona de Castilla. Aun en la primera mitad del siglo XIV, no surge referencia alguna a buques castellanos designados con tal vocablo. Lo que he podido observar es el uso sistemático del apelativo carraca cuando los castellanos hacen referencia a un buque que los marinos mediterráneos llaman coca o coca bayonesa.

${ }^{17}$ CARBonell Relat, La "coca”, nave, cit., p. 51. En la Historia anónima de Jerusalén, de 1177. En la Historia del asedio de Damietta, escrita por Oliverio el ESCOLÁSTICO, de Colonia, que vivió entre 1170 y 1227 . En la Historia Orientalis, de Jacobo de VITRY, muerto en 1240. En el Memorial de los podestas de Regio, de 1218. O en el Estatuto de Marsella de 1253: “...pro nave qualibet et hysneca vel coca.. 
a guerrear a Tierra Santa $^{18}$. Tales periplos las darían a conocer - y eventualmente utilizar-. Las crónicas son las primeras fuentes en advertir este nombre en fecha tan temprana como es $1218^{19}$. El Llibre dels Feits recoge el uso de una coca bayonesa en 1230 por parte del caballero Ato de Foces en su intento de pasar a Mallorca en ayuda del monarca; no obstante, la transcripción de esta obra - de 1343 - hace cuestionable dicha posibilidad ${ }^{20}$.

Para documentos contemporáneos, ya hay que situarse en los confines de los siglos XIII y XIV. La primera mención es en Génova $-1292{ }^{21}$, y en la Corona de Aragón es de 1302 con la Lezda de Tortosa ${ }^{22}$, siempre en relación a la actuación de los lezderos en decenios precedentes.

${ }^{18}$ Durante el siglo XII se producen varias campañas navales de cruzados nordeuropeos hacia Tierra Santa. Las distintas versiones de la expedición de 1147 sugieren que se componía de unas 200 unidades. La alemana y frisona del año 1189 , organizada con motivo de la tercera Cruzada, de 55 unidades, mientras que en la de 1217 eran 212 . Dado que la coca es conocida en las costas flamencas desde siglos antes, y ante la magnitud de dichas empresas, ésta podría haber formado parte importante de ellas. Flotas que irán haciendo esçalas por todo el Mediterráneo cristiano, descubriendo de este modo las cocas a sus marinos. Así, la campaña de 1189 llega a Marsella en agosto de 1190, y la de 1217 recorrerá todo el litoral norte mediterráneo en su viaje a Tierra Santa, tocando puerto en Ibiza, Tortosa, Barcelona, Tolón, Niza, Génova, Pisa, Roma... Véase A. ORTEGA VIllosladA, Viajes a Flandes e Inglaterra. ¿Cabotaje o “recta via”?, pp. 232-235.

19“Templarii milites in quadam eorum Coca intrantes, fluvium transire volentes..." En Agustín JAL Nouveau Glossaire Nautique (révision de l'édition publiée en 1848). Revisión dirigida por Michel Mollat, Paris, Editions du CNRS, 1988, vol. 3, p. 296. Voz coca-ae, de la obra: Memorial des podestats de Reggio.

${ }^{20}$ Se considera un lapsus del copista de la crónica, que estaría pensando en las muy conocịdas cocas bayonesas. Si bien no deja de sorprender la exactitud con que el cronista recuerda los tipos navales usados tanto por Ato de Foces como por Rodrigo de Liçana, el otro caballero que quería pasar a Mallorca con una tarida, de las empleadas en lạ conquista, y dos leños.

Hay que recordar que en 1217 se había producido el paso al Mediterráneo de más de doscientos buques alemanes y frisones -en cuyas flotas las cocas constituirían un elemento fundamental-. Así pues, teniendo en cuenta que actualmente se cree que la vida media de estos buques era de unos quince o veinte años, es probable que algunos de ellos completaran su servicio en el circuito mediterráneo, por lo que el relator habría querido destacar este hecho movido por el éxito que la coca tenía en esos momentos: "E venc missatge d'Aragó que Don Ato de Foces e don Rodrigo Liçana venien a nós... E don Rodrigo Liçana feu nolieiar una tarida d'aquelles que havien estades al passatge de Mallorques e dos Tlenys altres que li aduguessen conduit. E la tarida era bona per adur los cavalls, e els altres llenys portaven șon conduit, e arribà a Pollença; e venc ab trenta cavallers ben aparellats de tot ço que mester havien d'armes e de conduit. E Don Ato de Foces llogà una coca d'estes de Baiona. E, quant foren en la mar, la coca faïa aigua molta, sí que per dos llocs o per tres ne traïen de l'aigua en calders poques e calafataven ab estopa tot ço que podien, sí que ja volgueren ésser en terra, en qualque lloc poguessen eixir primer, també en Catalunya com en Mallorques. E la coca en què venia Don Ato de Foces e Don Blasco Maça ab les companyes dels cavallers qui venien ab ells, hagué a tornar per forca de temps a Tarragona, car lo temps los hi aduix. E cuidaren perir tots, per co car la coca faila molta aigua e era vella, e a males penes pogueren-ne traure la roba e els cavalls, sí que, quant o hagren treit la coca s'obrí e trenca's tota sobre la mar..." (Llibre dels feits del rei En Jaume, Capitulo 104 Ed. Ferran SolDEVILA, Les quatre grans Cròniques. I. Llibre dels feits del rei En Jaume. Revisió filològica de Jordi BRUGUERA; Revisió històrica de M. Teresa FERRER I MALLOL, Barcelona, Institut d'Estudis Catalans, 2007, pp. 196-197).

${ }^{21} \mathrm{ASG}$, Notai, cart. $\mathrm{n}^{\mathrm{o}} 71$, fols $93 \mathrm{v}-94 \mathrm{r}$. En 1312 se constata la primera coca fletada por genoveses (hasta ese año en todos los contratos rumbo al Atlántico norte figuran sólo galeras) para transportar de Mallorca a Flandes un total de 1.600 cantarias de diversas mercancías. Notario Durante Domenicus - Osbergenus Obertus, fol. $150 \mathrm{v}, \mathrm{n}^{\circ} 1$. En DOEHAERD, Les Galères génoises dans la Manche et la Mer du Nord à la fin du XIII ét au début du XIVe s. "Bulletin de P'Institut Historique Belge de Rome", XIX, Bruxelles-Rome, 1938, pp. 5-76.

${ }^{22} \mathrm{AHCB}, \mathrm{B}$ XXIII, 2 (Lezda de Tortosa), f. 61 r: "Bernardum Laurentii senior, VII teste deponit de contradictione et quod naues tam de Barchinona quam de alio loco soluere contradicebant et quod quadam cocha noluit soluere leudam mercimoniorum ipsius coche leudariis requirentibus leudam... 
En las Baleares, la encontramos en $1299^{23}$ y, también, en $1302^{24}$ con el pleito entre el florentino Savino Romei y A. de Casano, baile de Mallorca en tiempos del rey de Aragón, al incautarse éste de unas mercancías de su propiedad -además de armas- y, una vez restituidas, no consentirle llevárselas a Génova en el buque (coca-nave) de Daniel Can ${ }^{25}$. La siguiente referencia es de 1311 cuando el propio Daniel Can compra 30 libras de madera, de la cortada por orden del rey, para construirse una coca ${ }^{26}$. En 1312 aparece en los archivos genoveses Petrus Marocellus que arrienda a Nicolaus Burgarus una coca para transportar 1600 cantarias de mercaderías de Palma a Brujas ${ }^{27}$.

Pronto los marinos insulares las aprestarán para sus desplazamientos hacia aguas atlánticas. Los registros de Reebuda de exida de Sarrains de 1314 dan cuenta de la partida de, al menos, tres cocas con destino a Anfa durante los meses de julio y agosto ${ }^{28}$. Asimismo, se consignan las salidas de trece naos frente a seis cocas, en las que embarcan sarracenos hacia el reino de Granada y las costas de Marruecos. En 1316 son nueve cocas y una nao; en 1318, siete cocas y tres naos; en 1320, seis cocas, cinco naos, dos leños y una galera.

\footnotetext{
${ }^{23}$ Patrullas castellanas bloquean el estrecho de Gibraltar e impiden el tráfico mercante con sus enemigos granadinos. En Marbella, una galera de Castilla requisa cierta coca cargada con trigo por ciudadanos insulares "....pro eundo Maiorice vel prouinciam vel apud Janua, ut fuerat promissum...., bajo acusación de abastecer a los nazaríes: ... Item, ponit quod homines dicte coche portabant et portauerunt frumentum quod erat in dicta cocha apud Almeria, qui est locus sarracenorum..." ARM, Suplicaciones n ${ }^{\circ} 1$, ff. 220 r-225 v.

${ }^{24}$ En 1306 se da en Mallorca un pleito por la reclamación de Elisenda, madre del mercader mallorquín Nicolás Tarida fallecido en 1296 en Lisboa. En él se hace mención a la compra de una coca, concertada por este mercader en Sevilla, para singlar a La Rochela: "Item, lo deiats demanar per lo dit sagrament lo dit Luqi a sí con lo dit Nicholau Tarida e (D)aniel de Latorra leuaren viatge per anar a la Rutxela els e sa compaya ab los ditz Nicholau e Daniel e ab.(...) dels ne si en Xibilia feren (cart)a de compayia e en qual cocha anaren ne quins esmerces hi meteren e quant costaren..." ARM, LR-2, f. $141 \mathrm{r}$.

${ }^{25}$ ARM, Suplicaciones no 2, f. 35 v: “...ad litus maris quod ipse Saluinus debebat ipsa portare apud Januam in nauis Danielis canis non credit..." Marino de origen ligur, pronto se naturalizará mallorquín. Las huellas de sus derroteros cubren la zona de Berbería, el Estrecho y la costa atlántica africana.

${ }^{26}$ ARM, RP-3035, f. 34 v: "Item, reeberem d'en Daniel can per I ${ }^{\text {a }}$ quantitat de fusta la qual li fo venuda a ops de fer I $\mathrm{a}^{\mathrm{a}}$ cocha, so es a saber, da quela fusta la qual fo taylada de manament del Senyor Rey de bona memòria la qual lo Senyor Rey mana esser venuda..............XXX libras"; 56r: "Item, munta la summa de la moneda que-1 dit $\mathrm{G}$. Dauoltre pagua a diuerses persones per la fusta que fo taylada de "manament del Senyor Rey, de bona memoria, per la cocha e per les tarides les quals mana fer...

27 “....in instrumento naulizacionis sive locationis quam ipse mihi fecit de quadam cocha sua pro deferendo pro me et meo nomine cantaria mille sexcenta de rebus et mercibus de Maionicis ad partes Bruges...". ASG Not. Durante Domenicus, Osbergenus Obertus, p. 150v, $\mathrm{n}^{0} 1$. En DOEHAERD, Les Galères génoises, p. 60.

${ }^{28} \mathrm{ARM}, \mathrm{RP}-3764$, f. 40 r. Es de destacar que en estas fechas se emplee la coca preferentemente para singlar a los puertos atlánticos de Marruecos. La consabida falta de rigor al inscribir el tipo de buque hace que, en este caso, el del ciudadano mallorquín Tomás Pagá, con destino a Almería -y por extensión a Yspania- y Ceuta, sea registrado indistintamente como coca y como nao -si bien predominando el vocablo cocha-.
} 


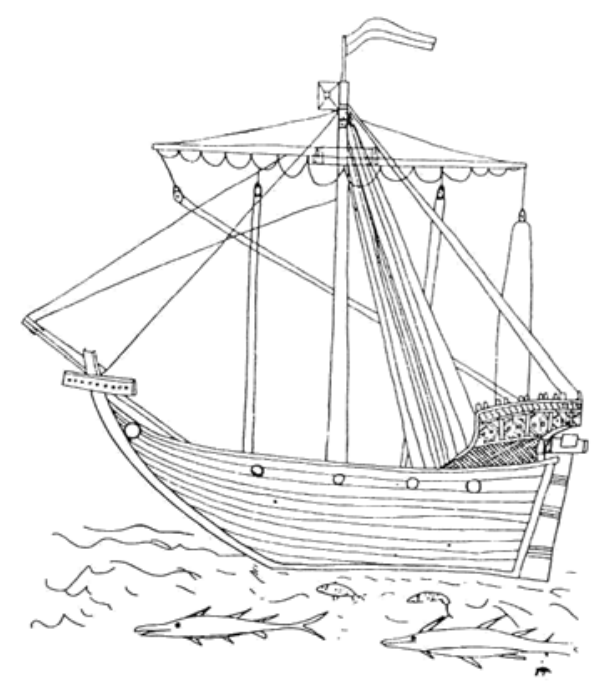

Coca mallorquina, de 1343, en el registro AH-3 del ARM

Los registros de Ancoraje posibilitan seguir - con las lagunas propias de estos volúmenes, pues sólo contienen datos relativos a 1321, 1324, 1330, 1332 y 1340 - su evolución en la marina mediterránea. Después de los leños, constituyen el grupo más numeroso que recala en el puerto de Palma. Es de destacar que mientras las naos - naus- desaparecen de las fuentes mallorquinas en 1321 como tal tipo de buque, las cocas alcanzan su máximo en 1321 y 1330 con un total de 66 entradas $^{29}$. En estos libros, donde se asientan las arribadas extranjeras, se observa que, en su mayoría, las cocas son - junto con las barcas - de castellanos y portugueses, y las galeras y taridas preferentemente de italianos.

\section{2. ÁREAS DE NAVEGACIÓN}

El estudio de algunos de los ordenamientos e imposiciones dados en Mallorca por Jaime III, y en la Corona de Aragón por Jaime II $^{30}$, Alfonso IV y Pedro el Ceremonioso nos revela la jerarquía en el uso con arreglo a su capacidad. El 18 de noviembre de 1330 se promulga en Mallorca un nuevo

\footnotetext{
${ }^{29}$ Cocas foráneas.

${ }^{30}$ Los fondos del ACA conservan la imposición otorgada el 14 de abril de 1315 por Jaime II, a petición de Barcelona, para salvaguardar sus costas del corso sarraceno. El control fiscal de las cocas difiere por número de cubiertas -2 ó 3 - y no se menciona el Atlántico. El génerico ultramar se reserva a las cocas/naves y leños (de bandas o grandes de una cubierta), circunscribiendo la ruta occidental al Estrecho -Spanya-. Por lo demás, las áreas de navegación anticipan las de 1341 .
} 
gravamen, sobre todos los productos, a fin de cumplir con las exigencias de Alfonso IV de completar una escuadra para la ineludible guerra con Génova. En un principio, la cooperación mallorquina implica la construcción de la mitad de los efectivos, si bien las quejas de los Jurados insulares disminuirían esta proporción. Ante lo inviable de instaurar otros impuestos, se decide aumentar la presión fiscal que, en el apartado naval, se refleja en los porcentajes a tributar.

Las cocas baleares navegan sin restricciones, a disimilitud de las catalanas y valencianas, constreñidas por la reglamentación de Alfonso IV. Las que se dirigen al extremo oriental del Mediterráneo abonan 15 libras por cubierta; hacia la franja comprendida entre Colliure y Aigües Mortes, la región de la actual Italia o la costa norteafricana, entre 4 y 5 libras; rumbo a puertos atlánticos: 8 libras si es hacia Cádiz o Sevilla, 10 libras al litoral africano y 15 libras si ponen proa a Inglaterra, Flandes o Brabante.

En 1331, Alfonso IV decreta sus conocidas ordenanzas marítimas ${ }^{31}$ en las que se pormenoriza, por tipo de buque y tamaño, los lugares de destino y el armamento a portar. Distribuye las cocas en tres niveles: entre 1.000 y 2.000 salmas $^{32}$, con autorización para desplazarse a Sevilla e incluso Flandes, pero costeando; de 2.000 a 3.500, o tres cubiertas, a Sicilia, Túnez, Flandes o Berbería. Las de más de 3.500 salmas eran las únicas que viajaban a ultramar - costa atlántica africana y Mediterráneo oriental-.

El mismo monarca ordena en Valencia - 1333 - una imposición, que recae sobre todo el comercio, con objeto de sufragar los gastos de las galeras comisionadas en el Estrecho. En ella también se prescriben las áreas de navegación: cocas de 1.000 quintales, o inferiores, podían ir hasta Venecia, Túnez, Nápoles, Pisa o Sicilia. Con mayor tonelaje se reservaban para la zona del Estrecho y, de allí, a Brujas o la costa africana, desglosándose la normativa en cuatro regiones: de Almería a Sevilla, más allá del cabo San Vicente, la costa meriní y la costa abbasí.

Por otra parte, la coca es el buque normalmente elegido por los marinos mallorquines para sus viajes oceánicos, como la Santa Cruz y la Santa Magdalena, cocas bayonesas en expedición ${ }^{33}$ a las Canarias, islas noueylament trobades... ....apellades illes de fortuna ${ }^{34}$.

Como todo bastimento medieval, era susceptible de presentar ligeras modificaciones. En 1331 fondea en Portopí un buque veneciano de los

\footnotetext{
${ }^{31}$ Publicadas por Josep M ${ }^{\text {a }}$ MADURELL MARIMON, Ordenanzas marítimas de 1331 y 1333 , “Anuario de Historia del Derecho Español”, XXXI (1961), pp. 611-628.

${ }^{32}$ En la reglamentación aquí comentada, la carga se mide siempre en quintales, salvo en este apartado de las cocas que será en salmas.

${ }^{33} \mathrm{ARM}, \mathrm{AH}-3$, ff. $10 \mathrm{v}-11 \mathrm{r}$ : “Con uosaltes haiats proposat ab les dites dues Coches armades anar e nauegar a les parts deles illes noueylament trobades ensus les parts de occident les quals illes vulgarment son apellades illes de Fortuna, e haiats elegit en Capità e president deles dites Coches e deles gents nauegants en aquelles lo dit Ffrancesch de Valers..."

34،...Com en G. Pere, ciutadà de Mallorches, patró d'una Cocha d'una cuberta, propona anar e fer uiage a les ylles noueylament trobades en les parts de ponent..." ARM, AH-3, f. 5 r.
} 
Casaleone que es descrito como coca de dos cubiertas y un corredor $^{35}$. Circunstancialmente adaptada para el traslado equino, en 1338 se embarcan en la del barcelonés Pedro Comte los del rey ${ }^{36}$. Al año siguiente, se pagaban 10 libras a Miguel Escolá, patrón de coca, por llevar de Mallorca a Nápoles el caballo del doncel Aries Ferrándiz ${ }^{37}$. En 1343, tras la conquista de Mallorca, Pedro IV ordena regresar a sus tropas, contando para ello con todo buque disponible, por lo que fletan al mallorquín Francisco Noguera su coca para pasar a Cataluña 21 caballos con sus jinetes y acompañantes. El embarque de los équidos se realiza introduciéndolos en una caja con la que izarlos a bordo ${ }^{38}$.

\section{TIMÓN BAYONÉS}

La especificidad que ha dado a la coca una cierta primacía en el estudio de la arquitectura naval del Medievo ha sido la inclusión del timón de codaste, fijado en el centro de la popa y accionado mediante la caña, en sustitución de los timones laterales o de espadilla ${ }^{39}$. La primera mención a las cocas bayonesas en el Mediterráneo la ofrece el cronista florentino Giovanni Villani, quien, haciendo referencia al año 1304, indica el paso a través del Estrecho de cocas equipadas con timón al codaste ${ }^{40}$. Hemos visto que desde mediados del XII se constata la entrada de buques atlánticos. Si tenemos en cuenta que las primeras informaciones sobre los timones de codaste son de finales del XII en Inglaterra, y que en sellos de la segunda mitad del XIII se representan cocas con estos timones, es difícil creer que no hubieran recalado

35“...duarum cohopertarum et unius corritoris que nunc est in Portu Pini...” ARM, AH436bis, $\dddot{\mathrm{f}} .11 \mathrm{r}$.

36"Primo, pagaren a·n Cipers Vila, lo qual estech e seruí per III meses en la Cocha d'en P. Comte, de Barchinona, hon anaren los Cauayls el arnès del Senyor Rey, a raó de VI libras lo mes..." ARM, RP-3050, f. 55 r.

37 “A·n Bernat des Soler, per pagar lo nòlit a·n Miquel Escolà, patró de coha, de portar lo cauayl qui fo d'en Aries Ferandis a Nàpols, axí con apar el dit libre de dades en cartes (LXXXVIII).......... libras". ARM, RP-3051, f. $40 \mathrm{v}$.

${ }^{38}$ ARM, RP-3055, f. 111 r: “..Primerament dissapte a XIII jorns de marc del ayn present, pagaren los dits procuradors a $n$ F F̈rancesch Nogera, ciutadà de Malorques patró de cocha, per nòlit de XXI cauayls e dels caualers e de lurs arneses e companyas... los quals portà de Malorques en les parts de Catalunya... .......... XXVIII libras, $V$ sous".

"Item, lo dia matex pagaren a $n$ Berenguer Barceló, mestre daxa, foren per una caxa gran que féu a ops de carregar los dits cauayls en la dita Nau con apar el dit libre de dades................. libras".

${ }^{39}$ Timones que continúan en uso a lo largo del XIV como en la coca de Guillermo Martí, de Aigües-Mortes: "Guillermus Martini de Aquis Mortuis, patronus cuiusdam Coche duorum timonorum, Johanes Egidii notxerius..." que obtiene, en 1359, guiaje en Mallorca para Berbería. ARM, AH, G-2, f. 10 r.

${ }^{40}$ Contemporáneo a estos hechos, en su Crónica de Florencia, Villani narra lo siguiente: "In questo medesimo tempo, certi di Bajona in Guascogna con loro navi, le quale chiamano coche, passano per lo streto di Sibilia, e vennero in questo nostro mare corseggiando, e feciono danno assai; e d'allhora innanzi i Genovesi e Viniziani e Catalani usaro di navicare co le cocche, e lasciarono ịl navicare delle navi grosse per piu sicuro navicare, e che sono di meno spesa; e questo fue in queste nostre marine grande mutazione de navilio" (Giovanni VILLANI, Cronica, tomo II, lib. 9, cap. 77). 
en el Mediterráneo hasta fecha tan tardía como 1304, cuando ya en 1277 marinos euromediterráneos frecuentaban los mares de Inglaterra y Flandes.

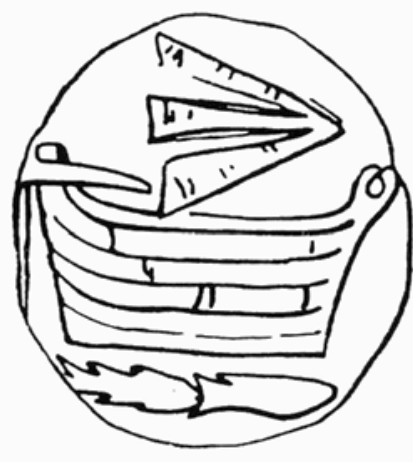

Coca de Stralsund, 1267

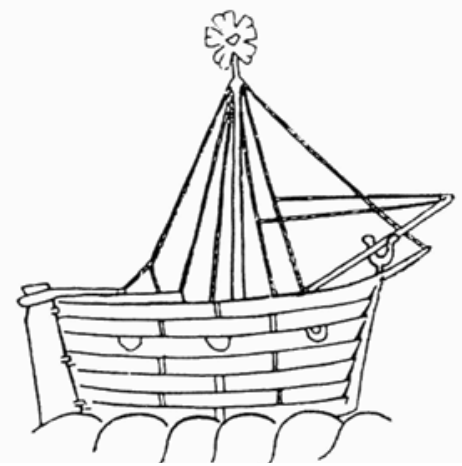

Coca de Harderwijk, 1280

Entonces, ¿a qué podía aludir Villani con esta afirmación? El rastreo en el Ancoratge de cocas castellanas de las zonas cántabra y vasca refleja una situación poco menos que sorprendente, ya que sólo figuran como cocas bayonesas en $1330^{41}$ y 1332 , algo que no sucede con anterioridad -1321 y 1324- ni en 1340. Si bien en el registro de 1324 aparecen gentes de Bayona, de las cuatro cocas anotadas ninguna de ellas, curiosamente, es calificada en Mallorca como bayonesa a pesar de reconocer la oriundez de los patrones ${ }^{42}$. Tampoco el denominativo bayonesa se cita en los libros de Eixida de sarrahins. El 21 de octubre de 1319 se documenta la presencia, en el muelle de Palma, del bayonés Arnaldo de Santo Luca, patrón de la coca Santo Espíritu, y de Pedro A., de San Juan de Luz, donde formalizan un préstamo de 30 marcas esterlinas de plata en croatas ingleses con Pedro de Segur y Martín de Guello, ambos de San Sebastián ${ }^{43}$, a devolver en dicha villa a

\footnotetext{
${ }^{41} \mathrm{El}$ mismo año, dos leños armados toman al abordaje, en aguas de Benidorm, una coca bayonesa de una cubierta que, después de expoliada, será hunḍida: "quadam cocham bayonesca unius cohoperte...vocata Sanctus Antonius... ...Ascenderunt in dictam cocham et abstraxerunt inde ea que ipsis placuit et post afondarerunt ipsam cocham que tum fuit efrondrata, sumersit seu iuit in profundum maris..." ARM, Suplicaciones $n^{\circ} 8$, f. 19 r.

${ }^{42}$ ARM, RP-1098, f. 5 v: “...Johan de Dercargi, de Bayona, entra VI yorns deguyn, coca........VIIIIsous"; (19v) "...Mateu Dorta, de Bayona, entra XXVI yorns duytubri, coca..........VIIIs".' (23 r): "Bartran Dalbinya, de Bayona, entra XV yorns de noembre, coca............VIIIIs". (30r): "Dimecres XXIIII yorns de gener reebem d'en Johan, dersen Johan de Bayona, per ancoratge de la sua coca quino pres albara d'entrada........... VIIIIs".

${ }^{43} \mathrm{El}$ easonense $\mathrm{P}$. Segura patronea en 1313 una coca de Xibilia rumbo, según presume el corsario valenciano Alfonso Guillem, a Mallorca con cierto numerario musulmán. ARM, LR-3, ff. $114 \mathrm{r}-\mathrm{v}$.
} 
Desidarer, esposa de Pedro de Limoges. Este buque es nombrado, en todo momento, simplemente como coca e incluso se emplea la expresión nauis siue coche $^{44}$.

El término bayonesa data de 1320, conforme se recoge en la declaración tomada en Cartagena a los testigos genoveses y lombardos residentes en Túnez cuando Ferrán González de la Marca, Lope García de Samarina y Juan de Guetaria, maestros de tres carracas - según la documentación castellana, o tres cocas según la mallorquina- arrasan el puerto el 12 de marzo de 1320. Durante el asalto queman una gran carraca - coca para los mallorquines - de tres puentes perteneciente al monarca tunecino y otras tres carracas con artículos prohibidos ${ }^{45}$. Los restantes buques son liberados, a excepción de la carraca del patrón mallorquín Fornells en la que fletan lo saqueado $^{46}$, y una tarida de bandas, genovesa, estibada con mercancía ilegal. En el estudio del pleito se observa que mientras los marineros de Daniel Can — todos ellos de procedencia mediterránea: baleares, ligures, lombardos...hablan siempre de $\operatorname{cocas}^{47}$, los testigos castellanos lo hacen de carracas $^{48}$. Los

${ }^{44} \mathrm{ACM}$, Protocolos notariales no 14807 , f. 33 v: "Johanes Fiyol, procuratore siue ministro Nauis siue coche domini Arnaldi de Sancto Lucha, de Bayona, vocate Sanctus Spiritus que nunc est in portu siue mollo ciuitate Maiorice ubi fuit et est detenta per venerabilem locum tenentem Maiorice; et Petrus Ricardi, de Sancto Johanne de Bayona, dispenserius eiusdem coche...

45 "Preguntado depues que fizieran los dichos cossarios, dixo que luego antel rrey que quemaran una grant carraca de tres cubiertas que era de y de Tuniz, et que quemaran otras cuatro carracas cargadas de madera e de trigo e de pez e otras cosas muchas deffendidas que en ellas auia". ARM, Pergaminos. Castilla-2 (declaración de miçer Giraldo).

${ }^{46}$ IbÍdem: “...ssi non una carraca de Mayorgas que era patrón della don Frenels, que vio que la affreytaron por diez mill torneses de plata pora yr al puerto de Cádiz o de Mayorgas. Et vio que la cargaren de aquellos aueres deffendidos que en el puerto de Túniz ganaron. Et otra tarida de Jenua que trayan cargada de trigo e de muchas armas e de mucho yerro et de mucha pez e de otras cosas muchas que enella ffallaron que eran todas las mas deffendidas ...".

47 “... significastis captiuem quam fecit armata Maioricarum de Cocha Danielis canis et aliis duabus Cochis Castellanorum in in(sic) portu Cartagenie..." ARM, LR-6, f. 17 v.

${ }^{48}$ Un aspecto a destacar es la ausencia de este vocablo en los registros mallorquines, no así en la documentación castellana. El estudio detallado de uno de los mayores pleitos mantenidos entre Mallorca y Castilla, como consecuencia del asalto que los corsarios castellanos realizaron en el puerto de Túnez a las cocas de los patrones Daniel Can y Fornells -y posterior respuesta mallorquina entrando en el puerto de Cartagena para no sólo recuperar sus buques y mercancías sino también llevarse los buques castellanos-, indica que el nombre de carraca no forma parte de la jerga de los marinos de Mallorca. En prolongado y laborioso litigio incoado en 1320, se enfrentarán por un monto de 200.000 doblas de oro, 1legando las apelaciones a la corte pontificia.

De este proceso judicial se han conservado las diligencias efectuadas por las partes, y si en todos los pergaminos castellanos se hace mención a los buques intervinientes -castellanos, mallorquines e incluso tunecinos- como carracas: "Preguntado depués que fizieran los dichos cossarios, dixo que luego antel rrey que quemaran una grant carraca de tres cubiertas que era de y de Tuniz, et que quemaran otras cuatro carracas cargadas de madera e de trigo e de pez e otras cosas muchas deffendidas que en ellas auía. Preguntado si enbargaron las otras carracas que auían dexado que non eran de su guerra, dixo que luego las soltaran e las mandaran yr a buena ventura con todo lo suyo, sinon una carraca de Mayorgas que era patrón della don Frenels que vio que la affreytaron por diez mill torneses de plata para yr al puerto de Caliz.(sic) o de Mayorgas et vio quela cargaren de aquellos aueres deffendidos que en el puerto de Túniz ganaron...", los testigos de origen mediterráneo - cónsules de Génova, Venecia, Sicilia, Cataluña y Mallorcá, a la sazón en el puerto de Túnez- sustituirán sistemáticamente este término por el de coca: "Micer Giraldo, mercador morador en Jennua, jurado e preguntado firmó e dixo por la jura que ffizo que el, estando en la villa de Túniz martes doze días andados del mes de março que agora passo, et que este dia grant mannana que ffuera en la villa de Túniz grant rroydo de aluoroço que cocas de bayoneses que auian quebrantado el puerto de Túniz et tomado todo quanto y fallaron en gisa que ffue el rrey a la rribera con muy grandes gentes a saber ssi lo podria deffender".

Estamos, pues, ante uno de los interrogantes que nos plantea el análisis de los buques medievales: el paralelismo de las expresiones carraca y coca. No deja de ser interesante observar 
corsarios aportan el testimonio de marinos, mercaderes y cónsules que se hallaban en el puerto de Túnez al desarrollarse los hechos. Éstos coinciden en nombrarlas como cocas de bayoneses: “...el, que estaua en la villa de Tuniz en esta quaresma que agora passo, un martes mannana que viera grand rroydo en la villa que cocas de bayoneses que auian quebrantado el puerto e muertos moros e cristianos quantos y fallaron, e que auian tomado quantas carracas e naues y auia et yua muy grand duelo en la villa..." 49 .

Teniendo presente que en los archivos de Bayona no ha quedado mención alguna a estas cocas, cabe preguntarse por el origen del apelativo bayonés o bayonesa. El timón de codaste, elemento inherente a estos buques, se documenta, ya a fines del XII, en el Atlántico norte y, siendo larga la tradición comercial de la costa septentrional peninsular y de la Gascuña - encabezada por Bayona - con esa zona atlántica, es probable que este tipo de timón fuera común durante el siglo XIII en la región de las Cinco Villas y el golfo de Vizcaya.

De los corsarios castellanos protagonistas de este litigio, dos -Ferrán González de la Marca y Lope García de Samarina- son oriundos de Castro Urdiales y el tercero - Juan de Guetaria- de San Sebastián y, sin embargo, los testigos los consideraban bayoneses. ¿Entendían los marinos mediterráneos como bayoneses a los tripulantes de las cocas del área marítima del golfo de Vizcaya, en lo que sería - para ellos- el golfo de Bayona señalando, de este modo, con el nombre del puerto más influyente de la región a una demarcación geográfica? ${ }^{50}$ Lo cierto es que, según los datos del ancoraje, las cocas

que la voz coca teniendo, como se sabe, ascendiente atlántico, no consta en los fondos castellanos, y que sería más lógico su uso en el norte peninsular que no en el Mediterráneo. Por otro lado, el estudio de este plẹito revela que, al menos conceptualmente, para los marinos de ambos mares poseían homóloga significación.

CARBONELL RELAT ( La “coca", nave, cit.,p. 47) admite que en el siglo XV carraca y coca eran semejantes y que tendrían un origen común, y se inclina por el ascendiente árabe - Harrâqalo que le induce a negar la posibilidad de que sean el mismo buque.

En el Archivo del Reino de Mallorca se preservan las dos partes del pleito. Las diligencias procesales practicadas por los corsarios de Castro Urdiales y San Sebastián se encuentran en los distintos pergaminos que componen la serie Castilla. La transcripción del litigio sustanciado en Mallorca corresponde a la signatura ARM, AH C-1760, ff. 91 r - 148 r. Actualmente estoy elaborando el estudio del mismo.

${ }^{49}$ ARM, Pergaminos, Castilla-2 (declaración de miçer Micoloso Lonbardo).

${ }^{50}$ Algo similar ocurre en el mar Negro. Los genoveses controlaban desde 1261 los puntos de abastecimiento de las rutas de Oriente, y Tana se había convertido en el mayor centro comercial ligur del mar de Azov, en la desembocadura del Don. En su primer atlas -del año 1313Vesconte se refiere a este mar como el mar de Tana: Hoc est mare tanna, dando el nombre de un puerto principal a toda una región geográfica. También AL-IDRISI comenta -en la primera parte del quinto clima- que el mar del golfo de Vizcaya era conocido por el mar de los ingleses: “...Desde el Castillo del Faro, ya mencionado, empiezan las montañas de Seba, que siguen la dirección dẹl mar, apartándose unas veces de él hasta un día de distancia y acercándose otras 15 millas, sin interrumpirse en toda su longitud, que cada una es de nueve jornadas de a 30 millas hasta la ciudad de Bayona, donde se unen a los montes del Templo de Venus. Estos montes limitan la península de España atravesando como un muro desde el mar Tenebroso o de los Ingleses al mar de Siria, entre Bayona y la tierra de Barcelona; y con el nombre de montes de las Puertas dividen los países de España y Francia”. (CCf. el texto de AL-IDRISI, en la colección "Textos Medievales", no 37, Valencia, Anubar Ediciones, 1974, pp. 78-79)

El 9 de junio de 1320, Sancho I reclama a Juan de Claramunt por la acción de los corsarios castellanos en Túnez, a los que describe como bayoneses: ". quod bayonenses ceperunt quandam taridam de bandis Leonardi de Podio, Januensis...” ARMM, LR-6, ff. 26 r. y 35 v-36 r. 
bayonesas son todas cántabras y vascongadas, no registrándose buques asturianos o gallegos bajo calificación semejante ${ }^{51}$.

\section{ARRIBADA DE PATRONES \\ DEL GOLFO DE VIZCAYA A MALLORCA}

El porcentaje de cocas - bayonesas o no- de la Hermandad que arriban al puerto de Palma es poco significativo en relación al total contabilizado. Así vemos que de las 66 cocas consignadas en 1321, sólo 2 son de esta zona (3\%); en 1324 de 61,12 (18\%); en 1330 de 68, 15 -de ellas 13 bayonesas- $-(22 \%)$; en 1332 de $49,17-10$ bayonesas- (34\%), año de mayor tráfico constatado de cocas de la Hermandad. Por último, en 1340 las encontramos en 2 de las escasas 40 entradas foráneas (5\%), aunque en Mallorca continuarán empleándose habitualmente e incluso se construyen en la ribera, tal es el caso de la coca bayonesa de una cubierta hecha en Palma, en 1341, en los aledaños del antiguo cementerio judío ${ }^{52}$. Estas cocas calificadas de bayonesas pagan de derecho 8 sueldos, es decir, como las cocas de una cubierta a las que se asimilan.

HERMANDAD DE LAS MARISMAS ${ }^{53}$

\begin{tabular}{|l|l|l|l|l|}
\hline \multicolumn{2}{|l|}{ CÁNTABROS } & \multicolumn{2}{l|}{ VASCONGADOS } \\
\hline AÑO & TIPO & PROCEDENCIA & TIPO & PROCEDENCIA \\
\hline 1321 & 2 cocas & $\begin{array}{l}\text { Santander (1) } \\
\text { Castro Urdiales (1) }\end{array}$ & -------- & ---------- \\
\hline 1324 & $\begin{array}{l}6 \text { cocas } \\
+1 \text { leño }\end{array}$ & $\begin{array}{l}\text { Santander (5+1L) } \\
\text { Castro Urdiales (1) }\end{array}$ & $\begin{array}{l}6 \text { cocas } \\
+2 \text { leños }\end{array}$ & $\begin{array}{l}\text { Bilbao (1) } \\
\text { San Sebastián } \\
(4+1 \mathrm{~L}) \\
\text { Fuenterrabía } \\
(1+11 \mathrm{~L}\end{array}$ \\
\hline
\end{tabular}

${ }^{51}$ Los marinos mediterráneos adoptarán este tipo de embarcaciones. En el registro de 1330 consta una coca bayonesa de Barcelona: “...Tomàs Soliuera, de Barcelona, entra XXXI yorns de mag, coca bayonesca, ay d'omes de Mayorcha la meytat, coca I ${ }^{a}$ cuberta...........IIs", y otra del tarraconense Juan Baró. ARM, RP-1102, ff. 6 v. y 21 v.

${ }^{52} \mathrm{ACM}$, Protocolos notariales $\mathrm{n}^{\mathrm{o}}$ 14564, ff. 147 r-148 r.: “...Sit omnibus notum quod ego Guillermus de Castro aulino, ciuis Maioricarum, dominus et patronus cuiusdam coche noue bayonesie unius cohoperte quam nouiter et, ex nunc, nuncupari volo Sancta Eulalia, adhuc existentis in suo esca et iuxta fossarum antichum iudeorum...

${ }^{53}$ Según los registros de ancoraje. Creada el 4 de mayo de 1296 con el objetivo de fomentar el comercio en la zona y terminar con las continuas querellas, participaban las villas de Santander, Laredo, Castro Urdiales, Vitoria, Bermeo, Guetaria, San Sebastian y Fuenterrabía. Pronto se enfrenta con la potente Bayona a la que excluye del trato mercante, vetando a su vez Bayona el comercio con Castilla. 


\begin{tabular}{|l|l|l|l|l|}
\hline 1330 & 4 cocas bay. & $\begin{array}{l}\text { Santander (3) } \\
\text { Escalante (1) }\end{array}$ & $\begin{array}{l}11 \text { cocas } \\
(9 \text { bay. }+2)\end{array}$ & $\begin{array}{l}\text { Vizcaya (1) } \\
\text { Bermeo (1 bay.) } \\
\text { Guetaria (4 bay.) } \\
\text { San Sebastián (3 } \\
\text { bay. }+1) \\
\text { Lequeitio (1 bay.) }\end{array}$ \\
\hline 1332 & $\begin{array}{l}12 \text { cocas } \\
(8 \text { bay. }+4)\end{array}$ & $\begin{array}{l}\text { Santander (3 bay.) } \\
\text { Escalante (1 bay.) } \\
\text { Castro Urdiales (4 } \\
\text { bay. }+4)\end{array}$ & $\begin{array}{l}5 \text { cocas } \\
(2 \text { bay. }+3) \\
+1 \text { leño }\end{array}$ & $\begin{array}{l}\text { Vizcaya (1 bay.) } \\
\text { Guetaria (1) } \\
\text { San Sebastián } \\
(2+1 L) \\
\text { Fuenterrabía (1 } \\
\text { bay.) }\end{array}$ \\
\hline 1340 & 1 coca & Santander & coca & Bermeo \\
\hline
\end{tabular}

Falta, no obstante, por elucidar la aserción tan taxativa que hace Villani al manifestar que fue precisamente en 1304 cuando la coca bayonesa produjo una convulsión en la marina mediterránea, año en el que la conflictiva situación política en la Península había mejorado.

En 1301 Fernando IV accede por fin a la mayoría de edad, y en 1303 la anarquía castellana es superada. El enfrentamiento con Aragón se reconduce y la tregua de junio de 1303, auspiciada por el monarca luso, devendrá en tratado de paz gracias a la sentencia arbitral de Torrella y los posteriores acuerdos de Ágreda, en los que se acoge también a los islamitas. Vemos, pues, que en la primavera de 1304 la Península goza de una tranquilidad con la que hacía tiempo no contaba.

Por su parte, las divergencias entre Francia e Inglaterra dan paso al cese de hostilidades con el refrendo, en junio de 1299, de la paz en Montreuilsur-Mer ${ }^{54}$, ratificada en el Tratado de París de 1303, por la cual se establece la paz perpetua y la libertad de comercio ${ }^{55}$. Desde este momento, las noticias sobre relaciones marítimas entre el Mediterráneo e Inglaterra y Flandes se incrementan considerablemente favorecidas por las disposiciones - conocidas como Carta Mercatoria- que Eduardo I promulga en febrero, en las que se otorga salvoconducto a mercaderes hispanos y portugueses para comerciar en Flandes. Pese a la conciliación, las desavenencias —en forma de corso-

\footnotetext{
${ }^{54} \mathrm{Da}$ inicio una época de paz que, exceptuado un breve periodo de tensiones en 1323-1324, se prolongará hasta 1337 cuando Felipe VI tome la Guyena y apoye las aspiraciones escocesas en contrapartida a las pretensiones de Eduardo III al trono francés, lo que provoca el arranque de la que se ha dado en llamar Guerra de los Cien Años.

${ }^{55}$ T. RYMER; R. SANDERSON, Foedera, Conventiones, Litterae et cuiuscunque generis Acta Publica inter Reges Angliae et alios quosvis Imperatores, Reges, Pontifices, Principes vel Communitates....Editio tertia. Apud Joannem Neaulme, 1765, vol. I, parte 4a , pp. 24-29:“....Est fait desores et afferme certaine pleine, enterrine et estable pees a durer perpetualment a touz jours, por eux, por lor heires et pur lor successors...
} 
persisten y el mallorquín Guillermo Pierre sufre las consecuencias al ser abordado, en 1304, en aguas de Saint-Mathieu ${ }^{56}$.

Y es que la cuestión flamenca distanció a ambas monarquías. A partir de 1292 se recrudecen las disputas entre los marinos ingleses del golfo de Vizcaya y los del mar del Norte, lo que suscita la reacción de Felipe IV proclamando felón a Eduardo I y confiscándole -en 1294- la Aquitania (conquistada prácticamente en su integridad entre 1294 y 1296). Para ello, se prevale del levantamiento en Gales y, además, crea un segundo frente al concertar una alianza con el monarca escocés Juan Balliol. Eduardo I responde decretando el embargo de la lana flamenca -1294- y abriendo al capeto un nuevo campo de batalla en el continente al coligarse en 1297 con el conde de Flandes Gui de Dampierre, en pugna con el francófilo conde de Hainaut por el control flamenco ${ }^{55}$. El armisticio impuesto en 1298 por Bonifacio VIII (seguido por el Tratado, 1299, de Montreuil-sur-Mer y la Paz de París de 1303) no aquietarán el territorio, ya que el intervencionismo de Felipe IV y la detención, en 1300, de Gui de Dampierre, determinarían el estallido de la revuelta de Brujas y la derrota francesa -1302- en Courtrai.

Reorganizar el ejército franco supuso desmedidos esfuerzos y la colaboración de contingentes extranjeros. En 1304 se reinicia la contienda y Felipe IV pasa con sus tropas a Flandes, nombrando almirante al genovés Raniero Grimaldi quien se presenta con 16 galeras con las que: “...corseggiarono e fatta gran guerra a le terre marine di Fiandra e preso molto navilio con mercatantia di Fiamminghi..."58. La réplica inglesa en apoyo a los flamencos bien pudo incluir el mandato a sus súbditos de la Guyena, con Bayona a la cabeza, de programar una expedición de castigo en el Mediterráneo contra los intereses de Génova: “...certi di Baiona in Guascogna, co.lloro navi, le quali chiamano cocche, passarono per lo stretto di Sibilia e vennero in questo nostro mare corseggiando, e lecirono danno assai..."59 ya que las cocas transfretaron el estrecho de Gibraltar como corsarios y no como piratas, es decir, respaldados por la oportuna licencia del

${ }^{56}$ PRO, C 66/124/ M. 4D: “...Ex graui querela Guillelmi Pierre, mercatoris de maioricis, et Domingi Peris, mercatoris de Seuilla necnon ex testimonis dilecti et fidelis nostri Johanes, Ducis Britannam, per literas suas patentes accepimus quod cum ipsi quandam nauem de Seuilla apud Seuilla bonis et mercimonis suis diuersis, ad valentiam quatuor milium et ducentarum librarum turonensis, carcari fecisent et iidem mercatores nauem illam cum bonis et mercimonis suis usque ad terram nostram Anglie ad negociandum ibi de eisdem duxisse voluissent...

${ }^{57} \mathrm{El}$ mayor problema derivaba de la relación entre los poderes aristocráticos, en general profranceses, y la influencia política de las ciudades dominadas por un potente artesanado cuyos intereses estaban vinculados al comercio exterior y, cada vez más, a Inglaterra, en la que tenían el principal abastecedor de lana.

${ }^{58}$ Giovanni VILlANI, Cronica, II, lib. 90 , cap. 77. En la batalla naval del genovés contra los flamencos concurren, por parte Guido de Dampierre: “...LXXX navi, overo cocche, al modo di quello mare, fornite con castella per battaglia, e in ciascuna il meno $\mathrm{C}$ uomini fiaminghi e del paese..." Finalmente la victoria era para las galeras ligures que apresarán la coca de conde flamenco.

${ }^{59}$ Ibídem. 
monarca inglés ${ }^{60}$, ocasionando en el Mediterráneo, según confesión de Villani, grave quebranto a los genoveses.

Fecha de recepción del artículo: noviembre 2007.

Fecha de aceptación y versión final: febrero 2008.

${ }^{60}$ Tuvo que ser un ataque selectivo ya que no se ha localizado referencia archivística alguna que mencione la presencia bayonesa en aguas mediterráneas antes de 1319, cuando la Santo Espíritu, coca propiedad del bayonés Arnaldo de Santo Luca, fondea en el puerto de Palma con mercaderes de San Sebastián. 